\title{
Energy and Throughput Analysis of Reservation Protocols of Wi Media MAC
}

\author{
Muhammad Alam, Shahid Mumtaz, Firooz B. Saghezchi, \\ Ayman Radwan, and Jonathan Rodriguez \\ Instituto de Telecomunicações, Campus Universitário de Santiago, Aveiro, Portugal \\ email: \{alam, smumtaz, firooz, aradwan, jonathan\} @av.it.pt
}

Received: May 6, 2013; Accepted: September 24, 2013

\begin{abstract}
WiMedia standardized a fully distributed high data rate communication using ultra wideband (UWB) for wireless personal area networks (WPANs). WiMedia Medium Access Control (MAC) provides a synchronous data communication service by Prioritized Channel Access (PCA) and isochronous service by Distributed Reservation Protocol (DRP). In this paper we propose three different approaches to analyze the reservation protocols, PCA and DRP, of WiMedias MAC. In the first two approaches we kept the portions of the superframe fixed for DRP and PCA traffics while the third approach follows random reservation. To test out the PCA and DRP by proposed approaches we presented a scenario of connected devices and incoming devices with mix traffic (video, VoIP and best effort). By means of simulations using Omnet ++ and analytical results, we demonstrate that the network offers maximum throughput as well as minimum blocking and energy consumption when using the third approach, provides access to medium by Hard, Soft and PCA reservation under the WiMedia rules and policies. The throughput fairness is also estimated for the three approaches using Gini index.
\end{abstract}

Keywords: Energy Efficiency, Distributed Reservation Protocol (DRP), Ultra-Wideband (UWB), Wireless Personal Area Network.

Journal of Green Engineering, Vol. 3, 363-382.

doi: 10.13052/jge1904-4720.341

(c) 2013 River Publishers. All rights reserved. 


\section{Introduction}

Medium access control (MAC) protocols play an imperative role to handle the challenging quality of service $(\mathrm{QoS})$ provisioning issues by efficiently controlling the access and utilization of wireless channels. In July 2008, WiMedia Alliance and European Computer Manufacturers Association (ECMA) International announced a joint development relationship. Both partners agreed upon the development of specific standards, which will be conducted jointly and by mutual cooperation of the engineers of member companies. For high data rates wireless personal area networks (WPANs) the ECMA standard [1] defined Physical and MAC layers which offer a number of policies and control mechanisms to ensure the QoSprovisioning. Ultra-Wideband (UWB) offers a number of advantages over other short range technologies; e.g., high data rate, low power and precise positioning, which makes it more suitable for the WPAN. UWB also supports various data rates ranging from 53.3 to $480 \mathrm{Mbps}$ over distances up to 10 meters [2].

In general, MAC protocols can be contention free and contention based, and some proposed ones are a combination of both. Contention free protocols require more challenging mechanisms to handle access to the channel by competing devices with different traffic types. For this reason WPANs are designed centralized or decentralized. IEEE 802.15.3 [3] is an example of centralized approach where the devices form a cluster called Piconet, and a coordinating device, called Piconet Coordinator (PNC), manages all the members of the piconet. The PNC has the knowledge of the member devices and assigns the available Medium Access Slots (MASs) often based on Time Division Multiple Access (TDMA) approach. But the centralized approach has a number of limitations; e.g., QoS provisioning, scalability of the network and if the PNC fails the whole network goes down. Furthermore, the connectivity of multiple Piconets is costly in terms of inter-piconet interference and leads to degraded performance [4]. To address these problems a distributed MAC, which is based on multiband orthogonal frequency-division multiplexing (MB-OFDM), has been proposed by WiMedia Alliance [5] and ECMA [1]. Both IEEE 802.15.3 and WiMedia MAC are based on slotted superframe where each device reserves slots in the superframe, by using reservation protocols defined in the WiMedia standard.

In the aforementioned background, this paper addresses and contributes to a detailed analysis of DPR and PCA reservation protocols of WiMedias MAC both analytically and through simulation. Unlike other works, mixed traffic (video, audio and best effort) is considered in the analysis. Besides 
analyzing the throughput and fairness of the WiMedia MAC protocols, we also analyze the blocking probability and the energy consumption of WiMedia devices experienced during transmission, backoff and reattempts to access the channel.

UWBS MAC protocol analysis, with different parameters and approaches, has been reported in previous works both by analytical models and through simulations, but most of the previous works focus on one or two traits of the analysis. That is, previous works either consider DRP or PCA in the analysis or consider throughput and delay or single traffic e.g. video, audio or best-effort or just analyze the protocols via analytical models. MAC layer is modeled as queuing system by Markovian arrival process (MAP) and phase type distribution $(\mathrm{PH})$ and the throughput is analyzed with respect to traffic load in [6], but the authors only consider DRP, ignoring its types without considering the different traffic types. In [7], the authors presented a study on the delay performance of DRP channel access for Multi-band OFDM Alliance (MBOA) UWB MAC by a bi-dimensional Markov chain model, where one dimension represents queue size distribution and the other is for allocated slots. UWB based WPAN, where physical layer adaptive modulation and coding is coupled with the DRP and the delayed acknowledgement schemes at the link layer, has been studied in [8]. However, a proper simulation environment, types of reservation protocols and impact of traffic types on protocols are missing. In [9], reservations are based on traffic type and priority but lacking the analysis of different reservation types (DRP Hard, DRP soft and PCA). Two reservation algorithms, subframe-fit and isozone-fit are proposed in [10] to analyze the blocking probabilities in reservation process. An analytical model for DRP based on shadowing effect, to account for slow statistical channel variations, has been studied in [11]. The authors consider both hard and soft DRP, and have proposed a channel model to describe the dynamic behavior of the UWB shadowing channel at the packet level. On a first-come first-served basis a contention-free distributed protocol has been analyzed for delay and throughput fairness in [12], but the work lakes a comprehensive analysis on blocking and shadowing. A study focusing only on the delay performance of DRP under different reservation patterns and the dynamics of UWB shadowing channel has been presented in [13]. The authors have modelled the system as a discrete-time single server queue with vacation represented by the quasi-birth and death process to analyse the performance of DRP.

The rest of this paper is organized as follows: In section II we present an overview of the WiMedia superframe and DRP. In section III, we present the proposed approaches for analysis with a mathematical model for blocking 
and energy consumption. Section IV represents the proposed scenario and simulation setup followed by results and discussion in section V. Finally, we conclude the paper in section VI.

\section{Preliminaries}

This section presents an overview of the UWB superframe structure, DRP and Gini index.

\subsection{Superframe}

UWB MAC channel time is divided into superframes. The total duration of the superframe is $65,000 \mu \mathrm{s}$ and is composed of 256 MASs with duration of $256 \mu$ s for each MAS [1]. The superframe has further been divided into two main parts, a Beacon Period (BP) and a Data Transfer Period (DTP) as shown in Figure 1. In BP, each user transmits its own beacon frames which consist of a number of Information Elements (IEs). These IEs has timing and control information of users to access the channel in a fully distributed manner and synchronize. The beacon frames represent information about current users and a view of the network which helps the incoming user to identify empty beacon slots, occupy it and transmit its beacons. The beacon frames are also used to reserve MASs in the DTP.

\subsection{DRP}

The MASs in the DTP are reserved, modified or released via DRP or accessed via Prioritized Contention Access (PCA). DRP is used to reserve the MASs mostly for isochronous traffic or nodes that need guaranteed access to the medium, while PCA provides differentiated channel access to the medium similar to IEEE 803.11e. When a node wants to reserve MASs, for data

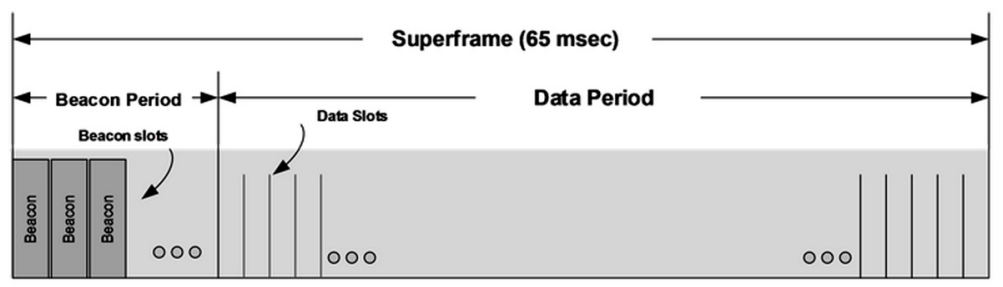

Figure 1 Superframe structure 
transmission or reception, it negotiates with its neighbors via DRP IE and reserves a set of MASs. The DRP frame contains a number of IEs representing different pieces of information. The DRP contains the control IEs which shows owner, status of reservation, reason codes, reservation types and some more information about the reservation conflicts [1]. The device that wants to start the reservation process is called the owner and the device that receives the information for reservation is referred to as target. The type of reservation can be Hard, Soft, PCA, Private or Alien BP. The Reservation status indicates the status of the DRP negotiation process which shows that whether a reservation is under negotiation, in conflict or established. The Reason Code is used by a reservation target which shows that whether a DRP reservation request was successful or not.

A DRP IE contains one or more DRP Allocation fields which are encoded using a zone structure. The zone structure is split into 16 zones numbered from 0 to 15 starting from the BP which are further divided into isozones - separated by fixed intervals. In this two-dimensional structure of the WiMediasuperframe each column of the superframe matrix is called an allocation zone as shown in Figure 2. In the reservation allocation IE, each node includes a Zone bitmap and a MAS bitmap, where Zone bitmap identifies the zones that contain reserved MASs and the MAS bitmap specifies which MASs in the zones identified by the Zone Bitmap field are part of the reservation. The reservation of MASs in the zones follows certain rules to ensure fairness; a detail is available in [1].

\subsection{Reservation Fairness using Gini Index}

The Gini index represents a measure of statistical dispersion developed by the Italian statistician and sociologist CorradoGini [14]. The Gini coefficient is a measure of the inequality of a distribution, 0 expressing perfect equality and a value of 1 expresses maximal inequality. We use Gini index to calculate the throughput fairness by the following formula. Let $l$ and $k$ be two users having observed throughput represented as $\mathrm{O}$ and the number of average throughput observed in the system is represented as $\mathrm{n}$ then the Gini index for throughput fairness is calculated follows.

$$
G=\frac{\sum_{k=1}^{n} \sum_{l=1}^{n}\left|\Theta_{a v-k}-\Theta_{a v-l}\right|}{2 n^{2} \overline{\Theta_{a v-R}}}
$$




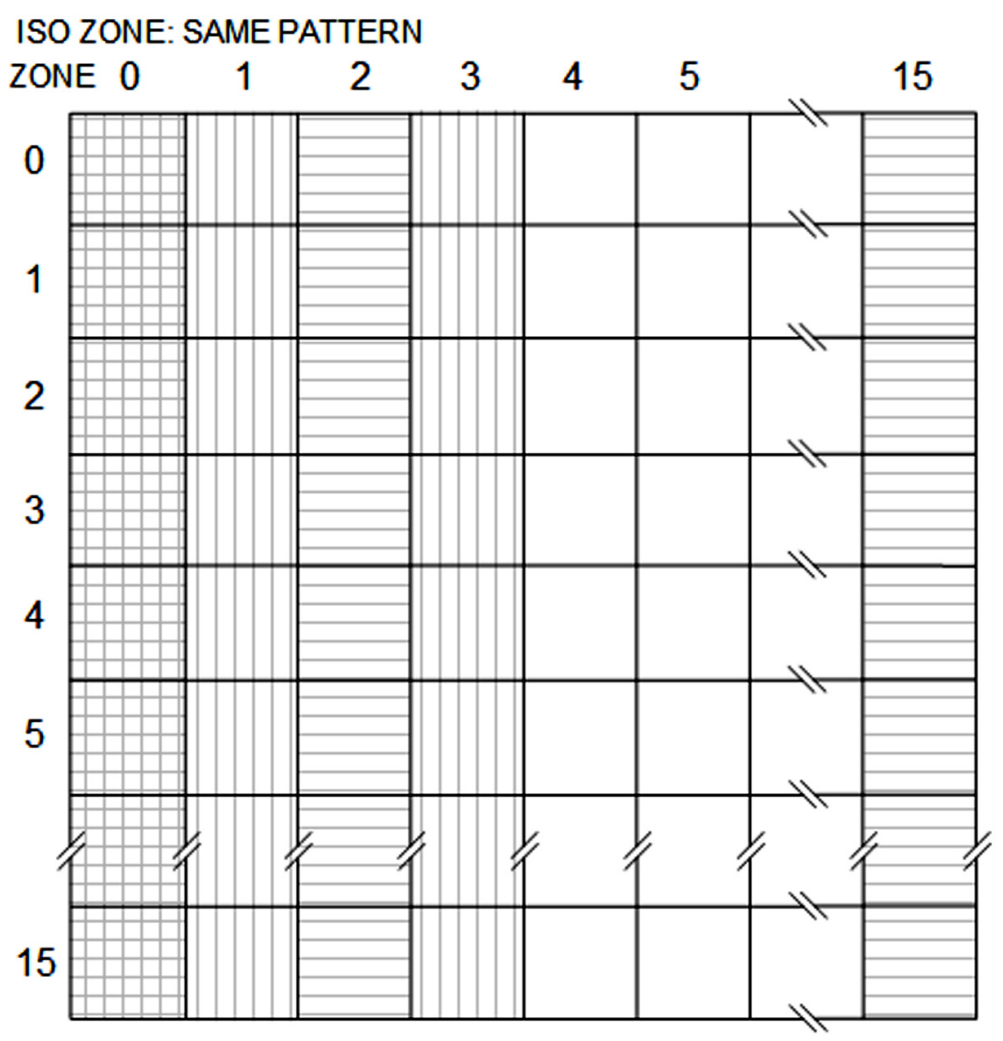

Figure 2 Two dimensional view of superframe

where

' $\Theta$ ' observed Average Throughput value of users ' $k$ ' and ' $l$ '.

' $n$ ' is the number of Average Througput oberved.

$\overline{\Theta_{a v-R}}$ is the Average Througput of all users placed within the coverage ' $R$ '

\section{Proposed Approaches for Analysis}

The DRP plays an important role to guarantee the QoS of isochronous traffic. The Devices use beacon IE to reserve the MASs in the superframe to transmit data. Since the MASs are allocated by DRP without prior knowledge of the traffic to ensure overall traffic load balance or fair distribution of the MASs among the nodes, we propose approaches to test the performance of the reservation based on the traffic types of incoming mobile nodes in the system. The 
nodes can use the DRP as a selfish approach to occupy the MASs for extended duration ignoring the waiting nodes or low priority traffic nodes such as best effort traffic. This selfish approach not only restricts the size of the network but also degrade the performance. Furthermore, the standard [1] defines a number of DRP reservation methods e.g, Hard, Soft, Private, PCA, etc. Hence, the MAS allocation need to be carefully handled during the beacon period and proper MAS access mechanism should be used.

\subsection{Analysis Approach 1}

In the first approach, we divide the superframe into two logically equal parts, DRP part and PCA part as shown in Figure 3. For isochronous traffic (audio, video), devices reserve the MASs based on hard DRP; once they occupy specific MASs they release it when their data finishes. The asynchronous data devices use the PCA part of superframe; devices occupy the MASs on first come first serve basis. Following are the assumptions for this approach:

- The devices can use only hard DRP.

- The isochronous traffic nodes cannot reserve the PCA MASs and PAC nodes cannot occupy the DRP MASs.

- The incoming devices are not allowed to use the relinquish request IE, once a MAS is reserved as Hard.

- The allocation of MASs is based on first come first serve basis.

The purpose of this approach is to check whether we can provide a balance to the MAS allocation by DRP and PCA and also to check the effect on PCA traffic in the presence of hard reservation for both types of traffic.

\subsection{Analysis Approach 2}

Since isochronous traffic needs more guaranteed channels, more MASs are reserved for DRP in this approach. Logically, one third of the superframe is

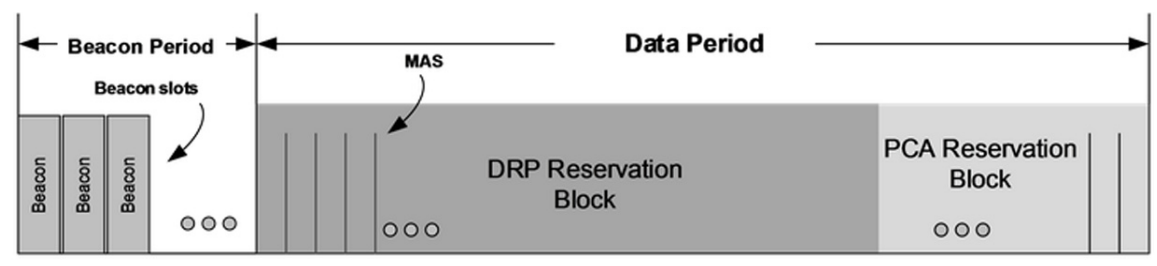

Figure 3 Superframestracture for approach 1 
reserved for PCA users and the rest for DRP users as depicted in Figure 4. This approach trails the following assumptions:

- Device can reserve MASs by both hard and soft reservation.

- Incoming users with isochronous traffic cannot reserve the PCA MASs and the same rule is applied to the PCA reservation.

- Incoming users can use the relinquish request IE to inform the neighbors to release the MASs occupied by soft reservation.

- The allocation of MASs is based on first come first serve basis.

The PAC or DRP reservation devices will go to backoff once they find a saturated superframe in their respective portions.

\subsection{Analysis Approach 3}

In this approach we use a dynamic reservation based on the traffic type and do not specify or fix the MASs for DRP and PCA reservations as shown in Figure 5.

This approach follows the following assumptions:

- No logical bounds are applied to the portions of superframe.

- Users can reserve MASs by hard and soft DRP and PCA reservations.

- The isochronous traffic devices can reserve the PCA MASs if they are available. If a device with isochronous traffic wants to enter the system, will first check free MASs, then will go to backoff and waits for a short duration for PCA occupied MASs to be released,and finally will request for releasing the MASs reserved by soft reservations.

- Incoming devices to system can use the relinquish request IE to inform the neighbors to release the MASs occupied by soft reservation.

- Devices that are connected and those which are entering into the system uses the DRP service primitive parameters e.g. desired bandwidth, available bandwidth and minimum bandwidth. The nodes use these parameters and estimate that how much bandwidth they need and how much is available in the system.

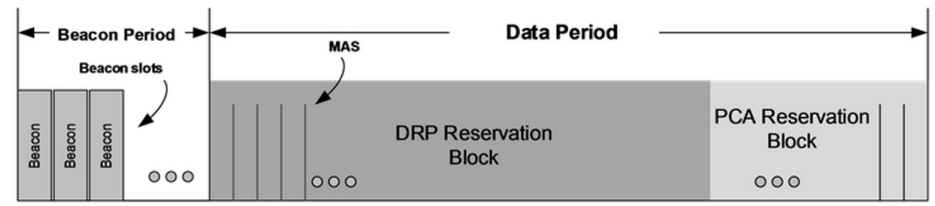

Figure 4 Superframe structure for approach 2 
Energy and Throughput Analysis of Reservation Protocols of Wi Media MAC 371

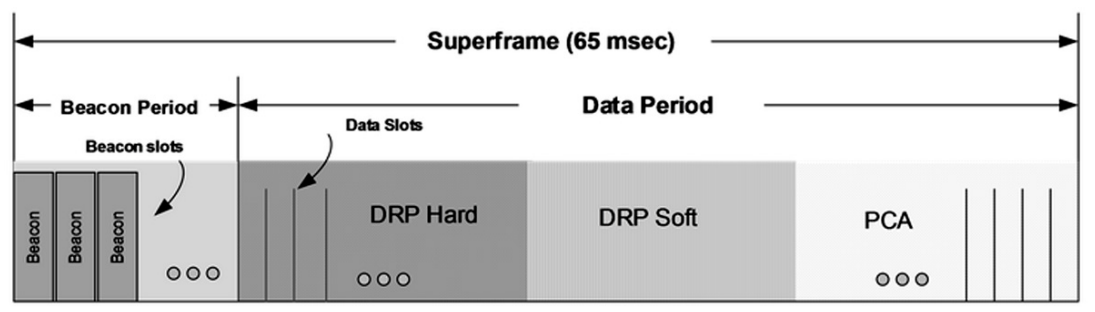

Figure 5 Superframe structure for approach 3

Although, the first two approaches are not fulfilling WiMedias standard rules and policies, we use them as baseline for the third approach to fully analyze the different reservation methods. Besides helping in analyzing the third approach, these approaches provide an overview of the reservation process and an impact of hard DRP on soft DRP and PCA.

The joule per bit energy and blocking probability of the above proposed approaches are modelled as follows.

For assignment of the MASs to PAC and DRP reservation, we have divided the superframe into $\mathrm{C}_{S F}$ number of MASs. Let $\mathrm{C}_{D R P}$ the number of current MASs reserved for DRP and $\mathrm{C}_{P C A}$ be the number of current MASs available for PCA reservation then from this definition we have:

$$
C_{S F}=C_{D R P}+C_{P C A}
$$

For approach 1, the number of MAS in superframe is as follows:

$$
C_{D R P}=C_{P C A}=C_{S F}
$$

And for approach 2

$$
C_{D R P}=3 C_{P C A}=3 / 4 C_{S F}
$$

In order to calculate how much energy per bit a user consumes, we define the following definitions. For an arbitrary user $X$, let us assume that $C_{T}$ is the duration of one MAS and according to the standard its value is $256 \mu$ s and $b_{x}$ is the number of MASs reserved by user $X$ and $R_{X}$ is the offered physical transmission rate. Then, according to [7], the number of bits per superframe transferred by user is given as follows

$$
F_{X}=b_{x}{ }^{*} C_{T}{ }^{*} R_{x}
$$

Now, let $E_{X}$ be the joule per bit energy consumed by user $X$ which is

$$
E=P_{T} / R_{x}
$$


Where $P_{T}$ is the transmission power.

Thus, by equation 5 and equation 6 , joule per superframe energy, represented as $E_{X S F}$, is given by

$$
E_{X S F}=E_{X}^{*} F_{X}
$$

To calculate the blocking probability of a new user $X$ into the system as $B_{X}$, we define

$$
\begin{gathered}
f(c)=\operatorname{Pr}\left(C_{D R P}=c\right) \\
B_{X}=\operatorname{Pr}\left(C_{S F}-C_{D R P}<b x\right)=\sum_{i=1}^{b x-1} f\left(\mathrm{C}_{S F}-b x+1\right) \\
B_{X}=f\left(C_{S F}\right)+f\left(C_{S F}-1\right)+\ldots+f\left(C_{S F}-b_{X}+1\right)
\end{gathered}
$$

where $X=1,2, \ldots ., k$

From equation 8 and equation 3 the blocking probability of DRP using reservations by user $\mathrm{X}$ for approach 1 is calculated as follows.

$$
\begin{gathered}
B_{X}=\operatorname{Pr}\left(\frac{1}{2} C_{S F}-C_{D R P}<b_{X}\right)=\sum_{i=1}^{b x-1} f\left(\frac{1}{2} \mathrm{C}_{S F}-b x+1\right) \\
B_{X}=f\left(\frac{1}{2} C_{S F}\right)+f\left(\frac{1}{2} C_{S F}-1\right)+\ldots+f\left(\frac{1}{2} C_{S F}--b_{X}+1\right)
\end{gathered}
$$

From equation 8 and equation 3, the blocking probability of DRP using reservations by user $\mathrm{X}$ for approach 2 is calculated as follows.

$$
\begin{gathered}
B_{X}=\operatorname{Pr}\left(\frac{3}{4} C_{S F}-C_{D R P}<b_{X}\right)=\sum_{i=1}^{b x-1} f\left(\frac{3}{4} \mathrm{C}_{S F}-b x+1\right) \\
B_{X}=f\left(\frac{3}{4} C_{S F}\right)+f\left(\frac{3}{4} C_{S F}-1\right)+\ldots+f\left(\frac{3}{4} C_{S F}--b_{X}+1\right)
\end{gathered}
$$

\section{Scenario and Simulation Setup}

In this section, we present our scenario for simulation, in which we have a connected network of 6 nodes and incoming nodes have a constant mobility as 
shown in Figure 6. For testing the proposed approaches, we consider random arrival of nodes with different traffics in the system. The above scenario is simulated using Omnet ++4.1 [15], an open source simulator, and different results are generated for the analysis of DRP and PCA under the rules of the proposed analysis approaches. The connected network is composed of 6 nodes and the incoming nodes vary which will affect the existing reservation. The nodes joining the network have a constant mobility of $0.5 \mathrm{~m} / \mathrm{s}$ as we are using constant speed mobility model. All the nodes have a maximum transmission power of $1 \mathrm{~mW}$. The data rate is $480 \mathrm{Mbps}$ and the traffic type is mix (video, VoIP, best effort). The scheduling of the allocation process is maintained on first-come first-served bases. The duration of the time slots are $256 \mu \mathrm{s}$.

\section{Simulation and Analytical Results}

Initially when the system is not saturated, the incoming nodes with both types of reservation, in all three approaches, are accommodated. But with the arrival of new nodes, the probability of getting the MASs to transmit data decreases especially for DRP users. Figure 7 shows the amount of allocated MASs to DRP and PCA reservation for the proposed approaches. In case of approach 1, the allocated MASs to DRP nodes are less due to the hard reservation whereas MAS allocation is high to PCA traffic nodes because of large portion of superframe is assumed to be utilized by best effort traffic nodes. Approach 2 provides more flexibility to the isochronous traffic nodes by using both hard and soft reservation but still cannot reserve the free MASs maintained for

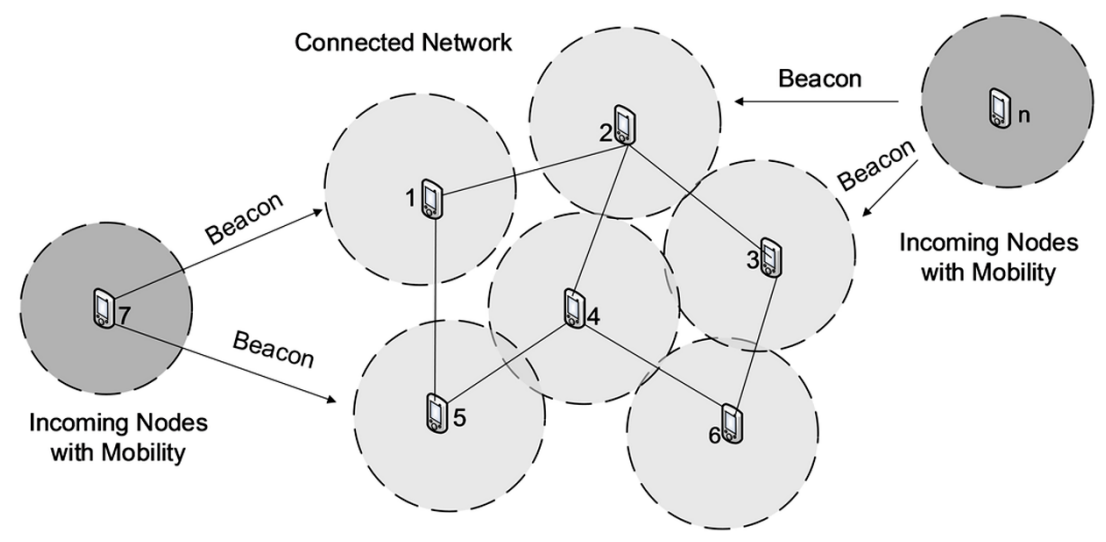

Figure 6 Network Scenario 


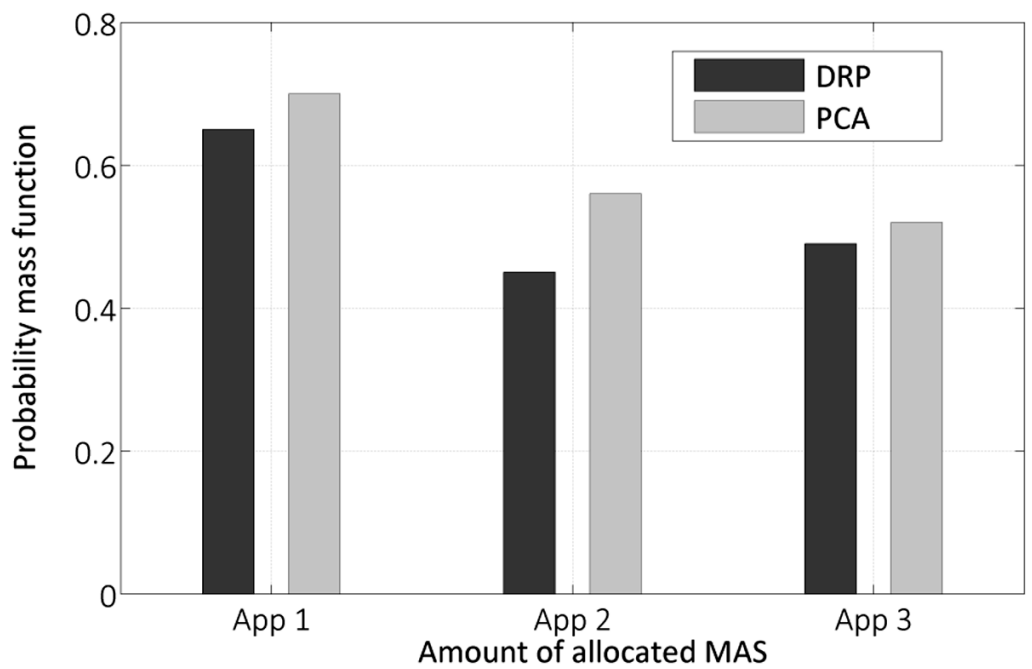

Figure 7 Allocation of MASs with DRP and PCA for the three proposed approaches for incoming users

incoming PCA reservations. The incoming node scans the reserved MASs and adopts its policy to reserve the MASs in a more flexible way in approach 3 and thus provides a more balance representation of MASs allocation to both isochronous and asynchronous traffic nodes.

Figure 8 shows the throughput of the system with the arrival of DRP and PCA traffic. Initially, the number of nodes in the system is less and there are free MASs available for incoming reservations. The proposed approaches contribute differently to the system overall throughput because of the different reservation types and rules. Approach 3 shows a high throughput because of soft reservation and PCAs MASs occupation. It reaches to 20 nodes high level and then remains constant because of saturation. The throughput of Approach 1 and 2 is low because of the fixed MASs for traffic types and depends on the traffic of joining node in the system. Therefore throughput of the system depends on the incoming node traffic type, rate and also the employed approach.

The number of packets in the system and its success and drop ratio depends on the flexibility of the reservation approach, rate of arrival, rules and policies applied to incoming traffic classes as well as the buffer size of the nodes. In case of hard reservation and keeping the superframe reservation portions fix for both traffic type leads to high packet drop. On the other hand, providing 


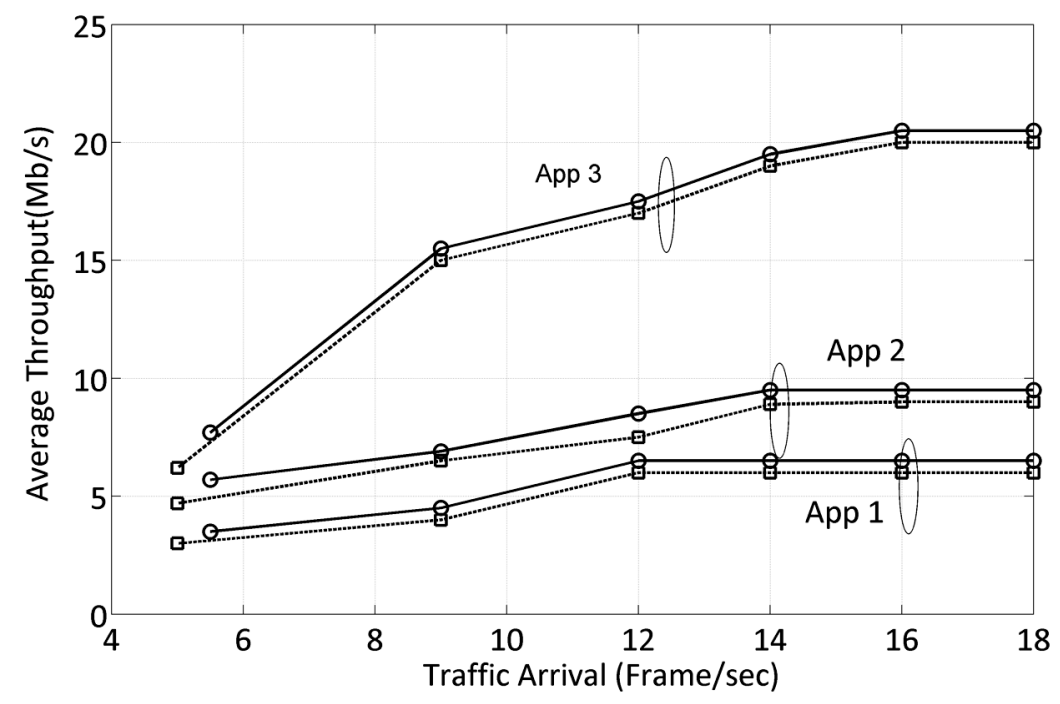

Figure 8 Throughput of DRP and PCA users for the proposedapproaches.

more flexibility to the reservation by utilizing the available MASs and keeping a balance between PCA and DRP hard and soft reservations the number of packet drops decreases. The packet drop of the incoming nodes for the three approaches is shown in the Figure 9.

For further insight, we provide the consumed energy of all the users both analytically and via simulations. The analytical results are illustrated by Figure 10. For our analysis, we assume that the amount of traffic, each user has to send, is equal, and it is represented by $B$. That is, $B_{1}=B_{2}=B_{3}=\ldots$ $B_{N}=B$ where $B_{x}$ is the traffic of user $X$. For our analysis, we assume that the value of $B$ is $10 \mathrm{Mb}$. We also assume that the transmission power $\left(\mathrm{P}_{T}\right)$ of all the users are constant and equal to $350 \mathrm{~mW}$. The energy consumed by user $X$ is calculated by $E_{x}=P_{T} \times T_{x}$ where $T_{x}$ is the time taken to transmit data $B_{x}$ at the gained data rate $R_{x}$ by user $X$. It should also be noted that users gain different data rates depending on their channel qualities. The total consumed energy by all the users is the summation of the energy consumed by each user; i.e, $E=\Sigma E_{x}$. In approach 1, for real-time traffics such as video and audio, the users utilize half of the superframe. Hence, on average, the data rates of the users are halved. This delays the transmissions, and results in higher energy consumption. In conclusion, the availability of more MASs and the introduction of soft DRP in approach 2 results in more available channels, less delay, and lower energy consumption compared to approach 1. 


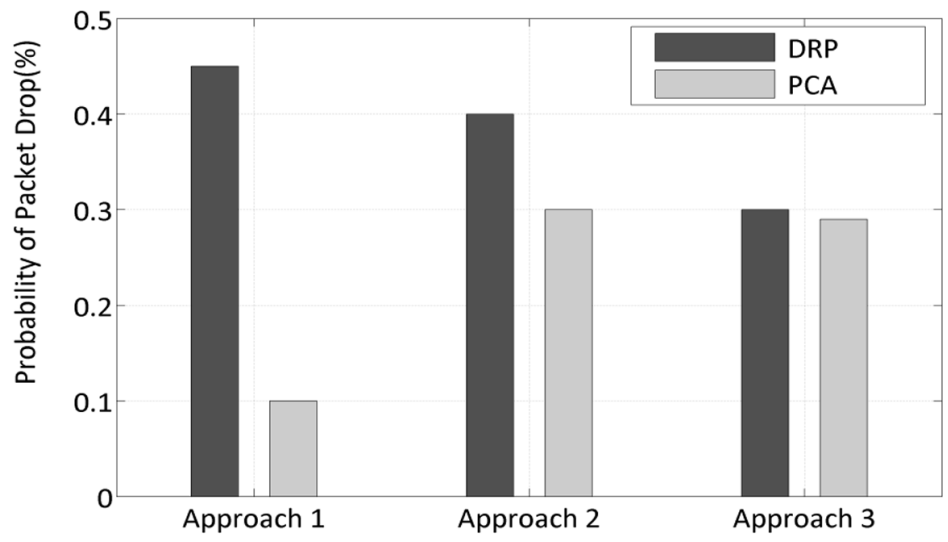

Figure 9 Blocking probality of the three appraoches

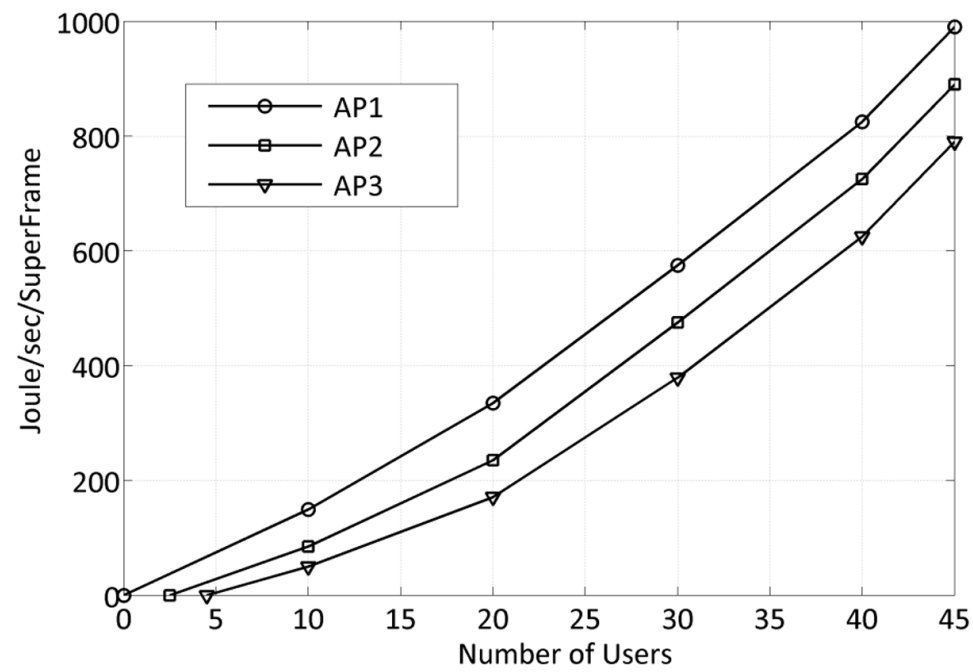

Figure 10 Gini index of allocated MASs

The simulation results for energy consumptions of the proposed approaches are shown in Figure 11. These results validate the theoretical results presented by Figure 10. The only important difference is that the simulations show higher energy consumptions than the analytical ones. This is due to the fact that, in our theoretical analysis, we do not consider the energy consumptions during the backoff stages and the reattempts to access the channel. 


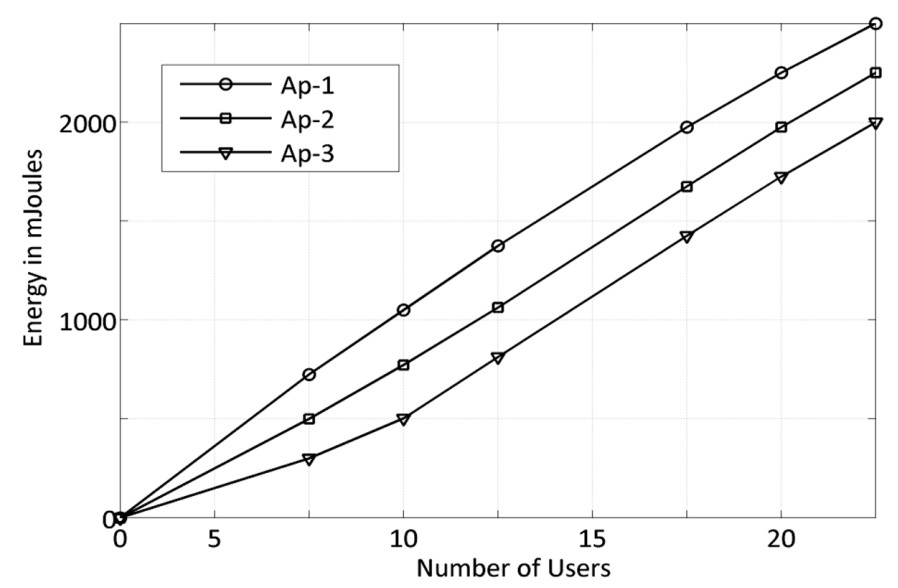

Figure 11 Simulation results for the energy consumptionof the three appraoches

The blocking probability of the three approaches for mixed traffic scenario is shown in Figure 12. The blocking probability of all approaches is low for a sparsely populated scenario due to sufficient MASs available for both DRP and PCA reservations. However, as the number of users increases, the blocking probability increases for all the approaches. In approach 1 , for video and audio traffic, the DRP users encounter frequent blockings due to fixed number of MASs as well as extensive use of hard DRP; the blocking probability reaches almost $90 \%$ once the number of incoming users to the system exceeds 60 . However, for the same approach, the PCA users utilizing sufficient MASs have less blocking probability. Approach 2, which offers flexibility to the DRP users, experiences less blocking compared to approach 1 due to sufficient MASs for DRP. The blocking probability of the PCA users is higher compared to approach 1 due to insufficient number of MASs for the PCA users. In approach 3 the incoming users have more flexibility to utilize the whole superframe when MASs are free.

The gini index is calculated using 30 nodes in terms of throughput according to equation 1. As shown in Figure 13, Approach 3 offers better fairness in term of throughput for all the nodes as MASs can be relinquished by utilizing soft reservation and also by estimation of number of available MASs for a specific incoming device compared to Approach 1 and Approach 2. Besides QoS, approach 3 also provides the quality of experience (QoE) to the users by avoiding unnecessary waiting in backoff and excessive re-attempting to access the channels, resulting improved system utilization. 


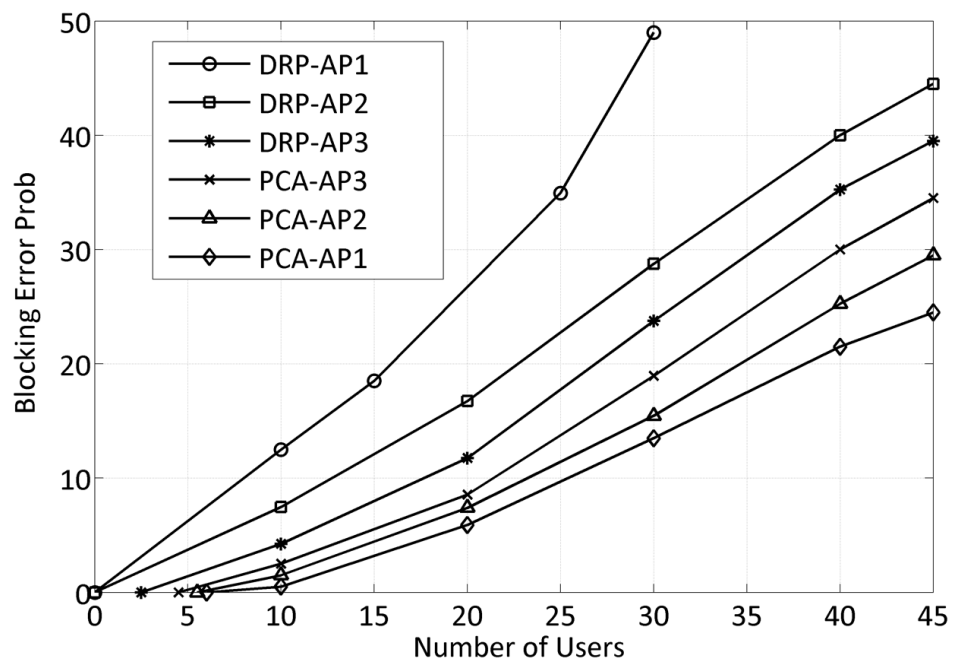

Figure 12 Blocking probality of the three appraoches

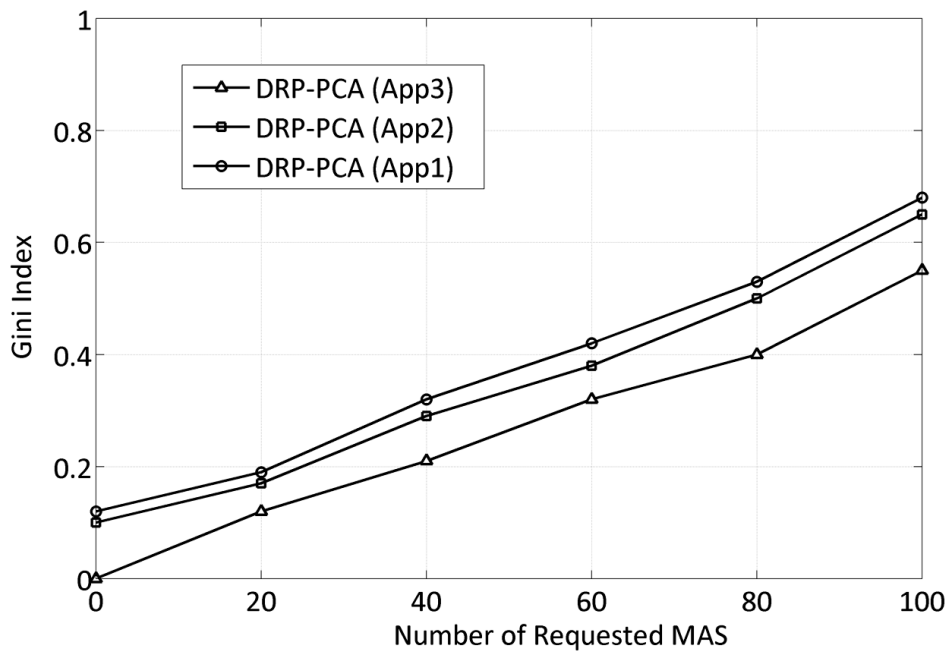

Figure 13 Gini index of allocated MASs

\section{Conclusions}

We proposed three different approaches to analyse the reservation protocol of WiMedia MAC for energy consumption and throughput fairness for both isochronous and asynchronous data traffic types. In approach 1, we kept the 
superframe half reserved for DRP hard and half for PCA utilizing users, while in approach 2, we introduced flexibility to DRP reservations for video and audio traffic by applying soft reservation and dedicating more MASs for DRP using nodes entering the existing network. To obviate the shortcomings of these two approaches, we proposed approach 3 which reserves the MASs using hard, soft and PAC reservation types with standard's bandwidth estimation primitives of ECMA standard. Furthermore, energy consumptions of the three approaches are also presented by using the different reservation approaches. The simulation and analytical results validate that approach 3 provides more fairness in terms of throughput, less packet drop and more energy efficiency compared to the other two approaches and thus not only guarantees QoS but also improves the QoE.

\section{Acknowledgements}

The research leading to these results has received funding from the European Community's Seventh Framework[FP7/2007-2013] under grant agreement number 248577 [C2POWER] and the PhD grant of the Fundaçãopara a Ciência e a Tecnologia (FCT) of Portugal, reference: SFRH / BD / 67027 / 2009.

\section{References}

[1] http://www.ecma-international.org/publications/standards/Ecma-368.htm, 3rd edition (December 2008).

[2] W. P. Siriwongpairat and K. J. R. Liu, Ultra-Wideband Communications Systems, 1st ed., New Jersey: John Wiley and Sons, 2007

[3] IEEE 802.15.3. (2003). Wireless medium access control and physical layer specification for high rate wireless personal area networks.

[4] L. X. Cai, X. Shen, and J. W. Mark, "Efficient MAC Protocol for Ultrawideband Networks," IEEE Communications Magazine, vol. 47, no. 6, pp. 179-185, 2009.

[5] WiMedia alliance. (2006, Dec. 15). WiMedia MAC Release Spec. 1.01. Distributed medium access control for wireless networks.[Online]. Available: http://www.wimedia.org/en/index.asp.

[6] N. Arianpoo, Y. Lin, V. W. S. Wong, and A. S. Alfa, "Analysis of distributed reservation Protocol for UWB-based WPANs with ECMA 368 MAC," in WCNC, 2008, pp. 1553-1558.

[7] H. Wu, Y. Xia, and Q. Zhang, "Delay analysis of DRP in MBOA UWB MAC," ICC '06. IEEE International Conference on Communications, vol. 1, pp. 229-233, June 2006.

[8] R. Zhang and L. Cai, "Optimizing throughput of UWB networks with AMC, DRP, and dly-ACK," in GLOBECOM 2008. IEEE Global Telecommunications Conference, 30 2008-Dec. 4 2008, pp. 1-5. 
[9] K. C. Go, J. H. Kim, S.-H. Oh, K. D. Moon, and K.-I. Lee, "Resource allocation algorithm considering a priority of service classes for Wimedia UWB system," in ICUIMC '09: Proceedings of the 3rd International Conference on Ubiquitous Information Management and Communication. New York, NY, USA: ACM, 2009, pp. 298-301.

[10] M. Daneshi, J. Pan, and S. Ganti, "Towards an efficient reservation algorithm for distributed reservation protocols," in INFOCOM 2010: Proceedings IEEE, March 2010, pp. 1-9.

[11] K.-H. Liu, X. Shen, R. Zhang, and L. Cai, "Performance analysis of distributed reservation protocol for UWB-based WPAN," IEEE Trans-actions on Vehicular Technology, vol. 58, no. 2, pp. 902-913, Feb. 2009.

[12] Bartke-Minack, K.; Guirao, M.D.P., "On perceived throughput and delay fairness of a distributed reservation protocol," Wireless Communications and Networking Conference (WCNC), 2011 IEEE, vol., no., pp.31-36, 28-31 March 2011.

[13] Kuang-Hao Liu; XueminShen; Ruonan Zhang; Lin Cai;, "Delay Analysis of Distributed Reservation Protocol with UWB Shadowing Channel for WPAN," Communications, 2008. ICC '08. IEEE International Conference on, vol., no., pp.2769-2774, 19-23 May 2008.

[14] http://en.wikipedia.org/wiki/Gini_coefficient (Last visited 10 January 2012).

[15] Varga, András. "The OMNeT++ discrete event simulation system."In Proceedings of the European Simulation Multiconference (ESM'2001), vol. 9, p. 185. sn, 2001.

\section{Biographies}

Muhammad Alam received his BS(CS) and MS(CS) degrees from the University of Peshawar and International Islamic University Islamabad, Pakistan, in 2005 and 2008 respectively. In 2009, he became a researcher at the Instituto de Telecomunicações - Aveiro, Portugal. He is a Ph.D. candidate and enrolled in MAP-i doctoralprogram Portugal and a member of the EU funded C2POWER research project. His research interests include green communications, cooperative networking, and energy efficient routing protocols for Wireless Mobile ad hoc Networks.

Shahid Mumtaz received his MSc. degree from the Blekinge Institute of Technology, Sweden and his Ph.D. degree from University of Aveiro, Portugal. He is now a senior research engineer at the Instituto de Telecomunicações Pólo de Aveiro, Portugal, working in EU funded projects. His research interests include MIMO techniques, multi-hop relaying communication, cooperative techniques, cognitive radios, game theory, energy efficient framework for $4 \mathrm{G}$, position information assisted communication, joint PHY and MAC layer optimization in LTE standard. He is author of several conference, journal and book chapter publications. 
Firooz Bashashi Saghezchi received the B.Sc. degree from Tabriz University (Tabriz, Iran) in 2000 and the M.Sc. degree from Shiraz University (Shiraz, Iran) in 2003 both in Electrical Engineering, Telecommunications. During 2004-2010, he served as a full-time faculty member at the Electrical Engineering Department of the Islamic Azad University, Garmsar Branch (Garmsar, Iran). He joined 4TELL Wireless Communication Research Group at Instituto de Telecomunicações (Aveiro, Portugal) in 2010 where he has been involved in several European research projects such as HURRICANE, C2POWER, and E2SG. He is currently pursuing his Ph.D. in MAP-tele Doctoral Programme in Telecommunications jointly at University of Minho, University of Aveiro, and University of Porto (Portugal). His research interests include game theory, cooperative strategies, energy efficiency, and cross-layer design for wireless networks.

Ayman Radwan received his BSc. degree from Ain Shams University (Egypt) in 1999; his Master of Applied Science from Carleton University, (Canada) in 2003 and his Ph.D from Queen's University (Canada) in January 2009. During 2009, he was a Research Associate at the Telecommunication Research Lab at Queen's University. In January 2010, he joined Instituto de Telecomunicações - Aveiro as a Senior Researcher. He is currently acting as the Technical Manager of the European research project C2POWER. His main research interests include radio resource management, quality of service provision, and performance evaluation in wireless and mobile networks, with emphasis on short range and cooperative communications. He is the author of several journal and conference publications as well as patents. $\mathrm{He}$ is an active IEEE member, acting as TPC member and reviewer for a number of respected conferences, journals, and magazines.

Jonathan Rodriguez received his Masters degree in Electronic and Electrical Engineering and Ph.D from the University of Surrey (UK), in 1998 and 2004 respectively. In 2002, he became a Research Fellow at the Centre for Communication Systems Research and was responsible for coordinating Surrey involvement in European research projects under framework 5 and 6. Since 2005, he is a Senior Researcher at the Instituto de Telecomunicações (Portugal), and founded the 4TELL Wireless Communication Research Group in 2008. He is currently the project coordinator for the Seventh Framework C2POWER project, and technical manager for COGEU. 


\section{M. Alam et al.}

$\mathrm{He}$ is author of more than 200 scientific publications, served as general chair for several prestigious conferences and workshops, and has carried out consultancy for major manufacturers participating in DVB-T/H and HSUPA standardization. His research interests include green communications, network coding, cognitive radio, cooperative networking, radio resource management, and cross-layer design. 